\title{
The importance of post-thaw subculture for standardizing cellular activity of fresh or cryopreserved mouse embryonic stem cells
}

\author{
Dong Woo Ko ${ }^{1,2, a}$, Jung Ki Yoon ${ }^{1,2, a}$, Jong il Ahn ${ }^{1,2}$, Myungook Lee ${ }^{1,2}$, Woo Sub Yang ${ }^{1,2}$, \\ Ji Yeon Ahn ${ }^{1, *}$, and Jeong Mook Lim ${ }^{1,2,3, *}$
}

\footnotetext{
* Corresponding Authors:

Ji Yeon Ahn

Tel: +82-2-880-4806, Fax: +82-2-874-2555,

E-mail: ahnji@snu.ac.kr

Jeong Mook Lim

Tel: +82-2-880-4806, Fax: +82-2-874-2555,

E-mail: limjm@snu.ac.kr

${ }^{1}$ Department of Agricultural Biotechnology, Seoul National University, Seoul 08826, Korea

2 Research Institutes of Agriculture and Life Sciences,

Seoul National University, Seoul 08826, Korea

${ }^{3}$ GreenBio Research Institute, Seoul National

University, Pyeongchang 25354, Korea

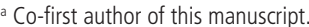

ORCID

Dong Woo Ko

https://orcid.org/0000-0001-7621-2534

Jung Ki Yoon

https://orcid.org/0000-0003-1436-7973

Ji Yeon Ahn

https://orcid.org/0000-0003-1792-1204

Jeong Mook Lim

https://orcid.org/0000-0002-2112-442X

Submitted Apr 17, 2017; Revised Jul 8, 2017; Accepted Aug 7, 2017

\begin{abstract}
Objective: Remarkable difference in cellular activity was found between early and late subpassaged embryonic stem cell (ESCs) lines, which can be created by subtle changes in cell manipulation protocol. This study subsequently examined whether post-thaw subculture of early subpassaged ESC lines could further affect the activity of the ESCs.

Methods: Fresh (as a control treatment) or cryopreserved F1 hybrid (B6CBAF1) early ESC lines (C57BL/6xCBA) of the $4(\mathrm{P} 4)$ or the 19 passage (P19) were subcultured once, twice or six times under the same condition. The post-thaw survival of the ESCs was monitored after the post-treatment subculture and the ability of cell proliferation, reactive oxygen species (ROS) generation, apoptosis and mitochondrial ATP synthesis was subsequently examined. Results: Regardless of the subculture number, P19 ESCs showed better $(p<0.05)$ doubling time and less ATP production than P4 ESCs and such difference was not influenced by fresh or cryopreservation. The difference between P4 and P19 ESC lines became decreased as the post-treatment subculture was increased and the six times subculture eliminated such difference. Similarly, transient but prominent difference in ROS production and apoptotic cell number was detected between P4 and P19 ESCs only at the 1st subculture after treatment, but no statistical differences between two ESC lines was detected in other observations.

Conclusion: The results of this study suggest that post-thaw subculture of ESCs under the same environment is recommended for standardizing their cellular activity. The activity of cell proliferation ability and ATP synthesis can be used as parameters for quality control of ESCs.
\end{abstract}

Keywords: Apoptosis; ATP; Cryopreservation; Embryonic Stem Cells; Mouse; Reactive Oxygen Species

\section{INTRODUCTION}

Development of cell manipulation technologies induces tremendous innovation of cell science and biotechnology, and cryopreservation-employed, cell bank further expands the feasibility of both basic research and clinical trials [1-5]. Standardization of cell bank and manipulation protocols and quality control are pivotal for minimizing batch effect and for yielding nonfluctuated results [5-8]. Likely to other cells, embryonic stem cells (ESCs) have been used for cell manipulation and cell banking [9-14]. To date, primary stem cell lines subcultured various times have been employed for various purposes without any concerns of batch difference and for the standardization, the cells of similar status (early, mid, or late passaged cells) have been employed in the same set of experimentation [15]. It has been reported, however, that a significant difference in cellular activity [16-19] was detected between early and late ESCs (39 vs 110 passage) [20-24]. It could further be possible that there might be a considerable difference even between the early ESC lines of different subpassge and that post-thaw 
subculture of the ESCs might make the difference prominently. Unfortunately, little consideration on the differences between the ESCs of the similar passages.

In this study, we examined whether cryopreserved, early ESCs of different passage had different cellular properties and subsequently inquired whether the results obtained from this experimentation could contribute to standardizing ESC manipulation protocols. Mouse primary ESCs of different passages (passage 4 or 19) were employed and cell proliferation, mitochondrial ATP production, reactive oxygen species (ROS) generation, and apoptotic cell numbers of the ESCs were monitored after being post-thaw subcultured different times.

\section{MATERIALS AND METHODS}

\section{Experimental design}

General characterization of the ESCs used in this study, which mycoplasma infection in cell manipulation system were concomitantly monitored, was universally conducted to know whether the ESCs being allotted into each experimental group had equal quality. Only primary ESC lines showing standard stem cell characteristics without mycoplasma infection were employed and early passaged ESCs at 4th (P4) or 19th times (P19) were either cryopreserved or not (fresh control). After treatment, they were concomitantly subcultured once, twice and six times. Cell proliferation assessed by cell doubling time (Dt), ATP synthesis, ROS generation and apoptotic cell number in the ESCs were monitored at the end of subculture. Randomized and controlled trials were conducted prospectively and all experiments were replicated three times. Comparisons were mainly made within fresh or cryopreserved groups by three manners; i) comparisons between P4 and P19 ESCs using accumulated values of the 1st, 2nd, and 6th subculture, ii) comparisons between P4 and P19 ESCs at the end of each culture, iii) comparisons among the 1st, 2nd, and 6th subculture within the same cells (P4 or P19).

\section{Experimental animal}

The review board of institutional animal care and use committee of Seoul National University approved our research (approval no. SNU-091028-4 and SNU-100825-3). All procedures for animal breeding and management, and surgery were followed by the standard protocols of Laboratory of Stem Cell and Bioevaluation, Seoul National University.

\section{Preparation of mouse embryonic fibroblasts}

Mouse embryonic fibroblasts (MEFs) were collected from 13.5-day-old fetuses of outbred Institute of Cancer Research (ICR) mice. The visceral organs, head and extremities of the fetuses were removed and the remaining tissue was cut into small pieces. The manipulated tissue were subsequently incubated in $0.04 \%(\mathrm{v} / \mathrm{v})$ trypsin-ethylenediaminetetraacetic acid
(EDTA) (Gibco Invitrogen, Carlsbad, CA, USA) for 6 min with agitation, and centrifuged at $110 \times \mathrm{g}$ for $2 \mathrm{~min}$. The supernatants were diluted in $10 \%(\mathrm{v} / \mathrm{v})$ fetal bovine serum (FBS; Hyclone Laboratories, Logan, UT, USA) containing Dulbecco minimal essential medium (DMEM; Gibco Invitrogen, USA). After being centrifuged at $390 \times \mathrm{g}$ for $4 \mathrm{~min}$ one more time, the pellets were seeded into plastic culture dishes containing DMEM for monolayer formation. When the fibroblasts formed a confluent monolayer, they were cryopreserved using $10 \%$ dimethylsulfoxide (DMSO; Sigma Aldrich, St. Louis, MO, USA). After thawing, MEFs were treated with $10 \mu \mathrm{g} / \mathrm{mL} \mathrm{mi-}$ tomycin C (Sigma Aldrich, USA) for $3 \mathrm{~h}$ and subsequently seeding in four-well multi-dishes (SPL, Pocheon, Korea)

\section{Collection of blastocysts}

B6CBAF1 embryos were generated by the mating of a female $\mathrm{C} 57 \mathrm{BL} / 6$ with a male $\mathrm{CBA} / \mathrm{Ca}$. Blastocysts were collected by uterine flushing of the females $72 \mathrm{~h}$ after mating. Collected blastocysts were washed in M2 medium consisting of 94.7 $\mathrm{mmol} / \mathrm{L} \mathrm{NaCl}, 4.8 \mathrm{mmol} / \mathrm{L} \mathrm{KCl}, 1.7 \mathrm{mmol} / \mathrm{L} \mathrm{CaCl}_{2} \cdot 2 \mathrm{H}_{2} \mathrm{O}$, $1.2 \mathrm{mml} / \mathrm{L} \mathrm{KH}_{2} \mathrm{PO}_{4}, 1.2 \mathrm{mmol} / \mathrm{L} \mathrm{MgSO}_{4} \cdot 7 \mathrm{H}_{2} \mathrm{O}, 4.2 \mathrm{mmol} / \mathrm{L}$ $\mathrm{NaHCO}_{3}, 23.3 \mathrm{mmol} / \mathrm{L}$ sodium lactate, $0.3 \mathrm{mmol} / \mathrm{L}$ sodium pyruvate, $5.6 \mathrm{mmol} / \mathrm{L}$ glucose, $20.9 \mathrm{mmol} / \mathrm{L}$ 4-(2-hydroxyethyl)-1-piperazineethanesulfonic acid, $1 \%(\mathrm{v} / \mathrm{v})$ penicillin/ streptomycin solution and $4 \mathrm{mg} / \mathrm{mL}$ bovine serum albumin before culture for ESC establishment.

Establishment and maintenance of embryonic stem cells The zona pellucida of the blastocysts was removed by acidTyrode's solution and, the zona-free blastocysts were subsequently seeded on MEF feeder layers in a gelatin coated four well multidishes containing knockout DMEM (kDMEM; Gibco Invitrogen, USA) supplemented with 3:1 mixture of FBS (Hyclone Laboratories, USA) and knockout serum replacement (Gibco Invitrogen, USA), 1\% (v/v) nonessential amino acids (NEAA; Gibco Invitrogen, USA), $0.1 \mathrm{mmol} / \mathrm{L} \beta$-mercaptoethanol (Gibco Invitrogen, USA), 1\% (v/v) penicillinstreptomycin solution and $500 \mathrm{unit} / \mathrm{mL}$ of leukemia inhibitory factor (LIF) (Chemicon, Temecula, CA, USA), this medium was used continuously for ESCs culture $[17,18]$. Colony-forming cells in the primary passage were subcultured after being trypsinized and general characterization to detect ESC-like cells was conducted after being subpassaged in LIF-containing medium. Subpassage was undertaken when the ESC-like colonies reached $80 \%$ confluency an average of 3 days intervals.

\section{Characterization of embryonic stem cells}

The ESCs used in this study were fixed in $4 \%(\mathrm{v} / \mathrm{v})$ formaldehyde (Sigma Aldrich, USA) at room temperature for $10 \mathrm{~min}$. After washing twice in phosphate-buffered saline (PBS), antibodies against Oct-4 (1:200 dilution; BD transduction, San Jose, CA, USA), stage-specific embryonic antigens SSEA-1 
(1:200 dilution; Santa Cruz Biotechnology, USA), SSEA-4 (1:200 dilution; Santa Cruz Biotechnology, USA), integrin a6 (1:200 dilution; Santa Cruz Biotechnology, USA) were used for marker staining. Localization of Oct-4, SSEA-1, SSEA-4, and integrin a6 was performed using the Alexa 488-conjugated anti-mouse antibody (Molecular Probes, Eugene, OR, USA), the Alexa 568-conjugated anti-mouse antibody (Molecular Probes, USA), Alex 488-conjugated anti-rabbit antibody (Molecular Probes, USA) and Alex 488-conjugated anti-goat antibody (Molecular Probes, USA).

To confirm spontaneous differentiation in vitro, ESCs were treated with $0.25 \%(\mathrm{v} / \mathrm{v})$ trypsin-EDTA (Gibco Invitrogen, USA), and the dissociated cells were subsequently transferred in LIF-free DMEM (Gibco Invitrogen, USA) containing 10\% (v/v) FBS (Hyclone Laboratories, USA). The embroid bodies (EBs) formed were seeded separately into four-well multidishes and cultured for 2 weeks. The EBs were fixed in $4 \%$ (v/v) formaldehyde (Sigma Aldrich, USA) at room temperature for $10 \mathrm{~min}$. The EBs were then stained with germ layer-specific markers: S100 (1:200 dilution; Abcam, Cambridge, MA, USA) for ectodermal cells; $\boldsymbol{\alpha}$-fetoprotein (1:200 dilution; Santa Cruz Biotechnology, USA) for endodermal cells; and Desmin (1:200 dilution; Santa Cruz Biotechnology, USA) for mesodermal cells. Antibody localization was performed using Alexa 488conjugated anti-rabbit antibody (Molecular Probes, USA).

Mycoplasma contamination test of embryonic stem cells Mycoplasma contamination of ESCs was confirmed by e-myco mycoplasma polymerase chain reaction (PCR) detection kit (iNtRON biotechnology, Seongnam, Korea) was used. Briefly, ESCs were treated with $0.25 \%(\mathrm{v} / \mathrm{v})$ trypsin-EDTA (Gibco Invitrogen, USA), and the dissociated cells $\left(5 \times 10^{5}\right)$ were used for genomic DNA extraction. The extracted genomic DNA was subjected to PCR amplification with primers for $16 \mathrm{~S}$ rRNA gene of M. fermentas. The PCR products were size-fractionated by $1.2 \%$ agarose gel electrophoresis and visualized by ethidium bromide staining.

\section{Freezing and thawing of embryonic stem cells}

After being trypsinized for $3 \mathrm{~min}, 2 \times 10^{6}$ trypsinized cells were placed into a $1.2-\mathrm{mL}$ cryovial containing $1-\mathrm{mL}$ cryopreservation solution consisting of $60 \%$ (v/v) DMEM (Gibco Invitrogen, USA), 30\% FBS (v/v) (Hyclone Laboratories, USA) and 10\% (v/v) DMSO (Sigma Aldrich, USA). A freezing container (Nalgene, Rochester, NY, USA) containing cryovials was placed in a $-80^{\circ} \mathrm{C}$ deep freezer for $24 \mathrm{~h}$, which kept a cooling rate of $-0.5^{\circ} \mathrm{C} / \mathrm{min}$, and plunged directly into liquid nitrogen. For thawing, the cryovials were placed in a $37^{\circ} \mathrm{C}$ water bath and the pre-warmed medium was added at the time of thawing [7].

\section{Measurement of cell proliferation}

Cell proliferation was measured by calculation of Dt. Briefly,
$5 \mathrm{~mL}$ of culture medium including $1 \times 10^{6}$ feeder layer cells were seeded on a $60-\mathrm{mm}$ culture plate. On next day, $5 \times 10^{5}$ ESCs were seeded and incubated in the $60-\mathrm{mm}$ culture plate. On day 3 of culture, trypsinized cells were counted with hematocytometer and the population doubling (PD) time was calculated following the formula [18]:

$$
\begin{aligned}
& \mathrm{PD}=\ln ^{(\text {final cell number/initial cell number) }} / \ln 2, \\
& \mathrm{Dt}=\text { time length }(\mathrm{h}) / \mathrm{PD} .
\end{aligned}
$$

\section{Measurement of ATP synthesis}

The intracellular level of ATP in ESCs was measured by luciferin-luciferase reaction kit (Sigma Aldrich, USA) [19]. Briefly, Ten thousands ESCs were placed into $100 \mu \mathrm{L}$ of ice-cold somatic cell reagent for $5 \mathrm{~min}$ at room temperature. Subsequently, $100 \mu \mathrm{L}$ of diluted ATP assay mixture solution buffer was added and incubated for $5 \mathrm{~min}$ at room temperature. After the solution being transferred into 96-well plate, luminescent reaction was measured by AutoLumat LB 953 Luminometer (EG\&G Berthold, Bad Wildbad, Germany).

\section{Measurement of reactive oxygen species}

The ROS generation by ESCs was measured by the 2,7' dichlorodihydro-fluorescein-diacetate (DCFHDA; Sigma Aldrich, USA) method originally described by Rosenkranz et al [20]. Two million ESCs were transferred to the culture medium containing $2.5 \mu \mathrm{g} / \mathrm{mL}$ DCFHDA for $10 \mathrm{~min}$ at $37^{\circ} \mathrm{C}$. After washing in DPBS, the treated cells were resuspended in 10 $\mu \mathrm{g} / \mathrm{mL}$ propidium iodide solution and subsequently loaded into a flow cytometry equipped with CaliburTM (Becton Dickinson, San Jose, CA, USA). The data obtained from FACS was numerically assessed using CELL Quest software (Becton Dickinson, USA).

\section{Detection of apoptotic cells}

To count the number of apoptotic cells in ESCs, one million cells were suspended in $100 \mu \mathrm{L}$ annexin $\mathrm{V}$ binding buffer and subsequently stained with FITC-annexin V and propidium iodide solution (apoptosis detection kit I; BD Bioscience, San Diego, CA, USA) for $15 \mathrm{~min}$ at room temperature. After staining, $400 \mu \mathrm{L}$ annexin $\mathrm{V}$ binding Buffer was added and subsequently analyzed by the Flow Cytometer (BD Science, USA) and the CELL Quest software (BD Science, USA).

\section{Statistical analysis}

A generalized linear model (PROC-GLM) in the Statistical Analysis System (SAS; Chicago, IL, USA) program was employed for evaluating model effects of each treatment. When statistical significances were detected by analysis of variance in SAS package, each treatment effect was compared using the least-squares method. Statistical significance in each compari- 
son was considered when p value was as less as 0.05 .

\section{RESULTS}

Preliminary experiment: ESC characterization and mycoplasma test

The ESCs provided for this study had standard characteristics compared with referenced mouse ESC lines. As shown in Figure 1, the established ESCs were positive for anti-Oct-4, antiSSEA-1, anti-integrin a 6 antibodies, but no reactivity was detected in SSEA-1 antibody staining. The ESCs were formed EBs, and the cells of the EBs stained positively for anti-S100 (ectoderm), anti- $\boldsymbol{\alpha}$-fetoprotein (endoderm), and anti-desmin (mesoderm) antibodies. Mycoplasma contamination was not detected in the ESCs and 16S rRNA gene of M. fermentas were not amplified in genomic DNA of the ESCs.

\section{Cell proliferation assessed by cell doubling time and} ATP synthesis

In both fresh (Figure 2) and cryopreserved (Figure 3) groups, P19 ESCs had shorter $(\mathrm{p}<0.0087)$ Dt and less $(\mathrm{p}<0.0176) \mathrm{mi}-$ tochondrial ATP production than P4 ESC. Such difference mainly resulted from the difference at the 1st subculture (Figure 2B, 3B). Similar Dt was detected among the 1st, 2nd, and 6th subpassage in both fresh and cryopreserved ESCs. Regardless of fresh or cryopreserved, dramatic $(\mathrm{p}<0.05)$ decrease in ATP production was detected in P19 ESCs, but not in P4 ESCs (Figure 2D, 3D). Likely to cell proliferation, however, the difference between P4 and P19 ESCs was gradually diminished as the subpassage was increased and no significant difference was detected at the second and sixth subcultures.

\section{Generation of reactive oxygen species}

Regardless of fresh or cryopreserved, significantly increase in ROS generation was detected in P4 ESCs as the subpassage was progressed, while decreased generation was in P19 ESCs (Figure 2F, 3F). As results, there was no significant model effect of ROS production between cryopreserved P4 and P19 ESCs when compared with overall values (Figure 3E). In fresh group, however, larger ROS generation was detected in P19 ESCs than in P4 ESCs, such derived from higher ROS level in the P19 at the 1st and 2nd subcultures. Although such generation pattern was different in both fresh and cryopreserved groups, the difference between P4 and P19 ESCs became reduced as the subpassage was increased.

\section{Number of apoptotic cells in the embryonic stem cells}

Regardless of fresh and cryopreserved groups, there were no statistical differences between the P4 and P19 groups (Figure $2 \mathrm{G}, 3 \mathrm{G}$ ). Similar level was detected in each subpassage (Figure $2 \mathrm{H}, 3 \mathrm{H}$ ), but in cryopreserved group, the apoptotic cell number was significantly decreased in P19 ESCs as the subculture was increased.

\section{DISCUSSION}

In this study, it was apparent that care should be taken to control the quality of the stem cells even that were maintained stably in vitro. Even cellular activity of early ESC lines (up to 20 times) became different by different times of post-thaw subculture. Little consideration on cellular difference was made in early primary stem cell lines subcultured different times and our results show that the difference caused by different cell manipulation (such as the frequency of post-thaw subculture) is responsible for batch effect and data fluctuation. Both fresh cell manipulation and cell banking program requires the standardization of cellular activity in each experimental treatment for yielding reliable data, and the standard operation protocol of the ESCs is extremely important.

Stable and homogenous, primary ESC lines of different passages were used in this study. The P19 ESCs had higher proliferative ability as compared with the P4 ESCs. Only 15 times difference in cell passage number yielded significant difference in cellular activity such as cell proliferation and mitochondrial generation, however, although the ESCs used had similar stem cell characteristics. Cryopreservation itself did not diminish the difference, but the difference was reduced as postthaw subculture of the ESCs was progressed. These results meant that cell passage of ESCs induces alteration of cellular properties and that more than twice of concomitant subculture greatly helped to reduce the passage-induced difference. Probably, post-thaw subculture of proper times under the same environment may be beneficial for becoming similar cellular activity of the ESC lines showing different activity. The results from the present study provide direct evidence of the subpassage effects on the cellular activity of ESCs. Increasing ATP generation in P4 ESCs did neither cause more ROS generation nor increase apoptotic cell number. Probably, increased ATP level stands for upregulated activity of cellular activity during very early passage for acquiring cellular homeostasis. Regardless of the feasibility of cryopreservation [25-27], freezing and thawing procedure alters cellular activity and induces genetic instability after freezing and thawing [28,29]. Subculture after thawing is therefore important for regaining cellular ability or for stabilizing genome activity [20,21,23,30].

Quality management system is necessary for managing various experimental protocols from stem cell manipulation, which greatly contributes to developing stem cell-based therapy, as well as to standardizing data quality. Standardizing of cell passage and pre-culture before main treatment is helpful for eliminating data fluctuation and for improving data quality, which can increase the completeness of various stem cell researches. The results obtained from this study contribute to establishing quality management system of the ESCs employing 
(A)
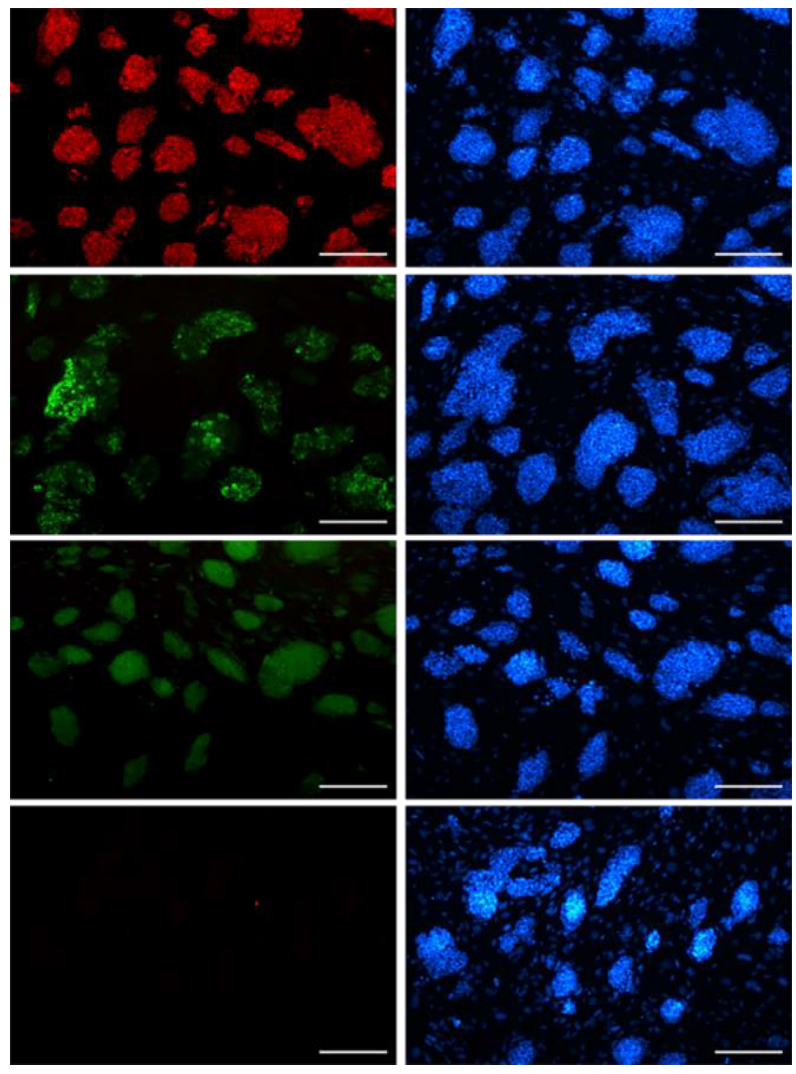

(B)
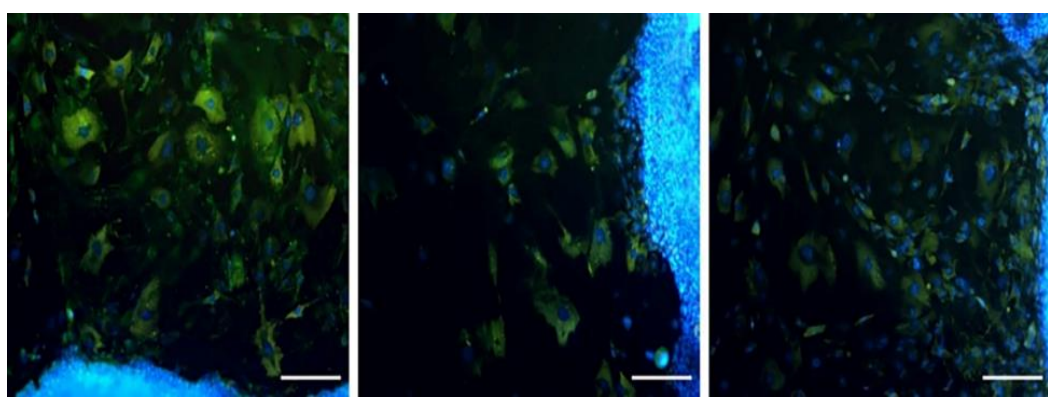

(C)

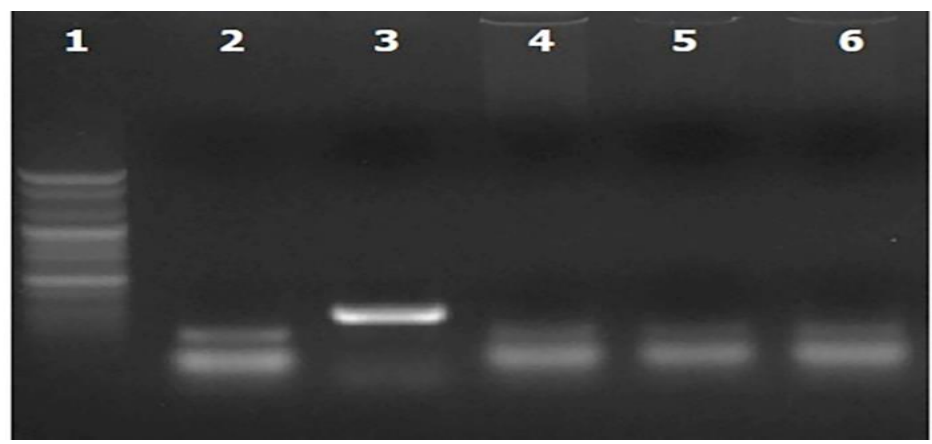

Figure 1. (A) Characterization of mouse B6CBAF1 embryonic stem cells (ESCs). The ESCs were provided for DAPI staining using anti-Oct-4, anti-SSEA-1, anti-integrin a6 and anti-SSEA-4 antibodies. The ESCs characterized were positive for Oct-4, SSEA-1 and integrin a6, while negative for SSEA-4. Scale bar = $200 \mu \mathrm{m}$. (B) Differentiation of mouse B6CBAF1 ESCs. The embroid bodies derived from ESCs were provided and the immunocytochemistry using anti-S100 (ectoderm-specific), anti- $\alpha$-fetoprotein (endoderm-specific), and anti-desmin (mesoderm-specific) antibodies was conducted for DAPI counter stain. The embryoid bodies were positive for all markers. Scale bar = $200 \mu \mathrm{m}$. (C) Test of ESCs for mycoplasma infection. PCR (polymerase chain reaction) was employed for the detection and non of ESCs was positive for mycoplasmosis. Lane 1, 100 bp ladder; lane 2, negative control (PCR without the addition of template); lane 3, positive control (genomic DNA from M. fermentans infected K562); lane 4, 5, and 6, ESCS. 


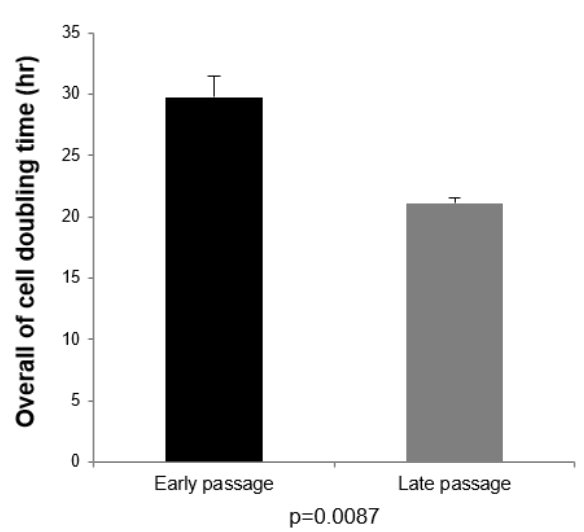

(A1)

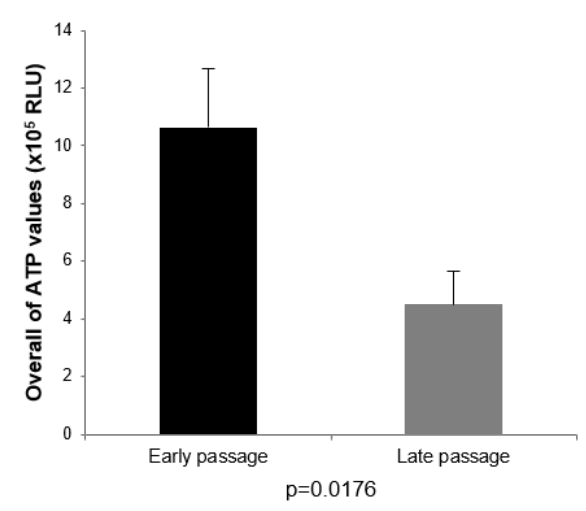

(B1)

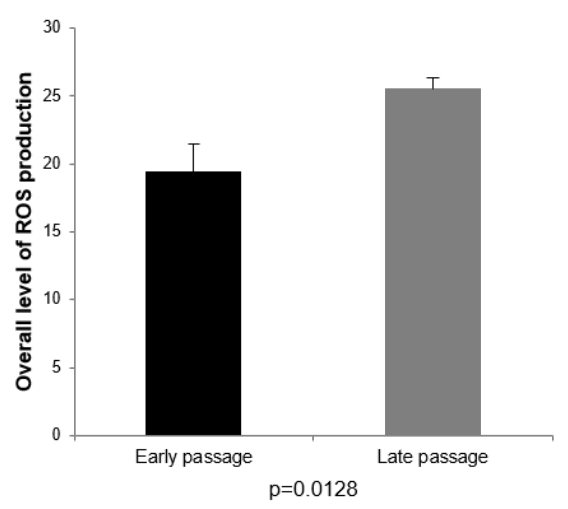

(C1)

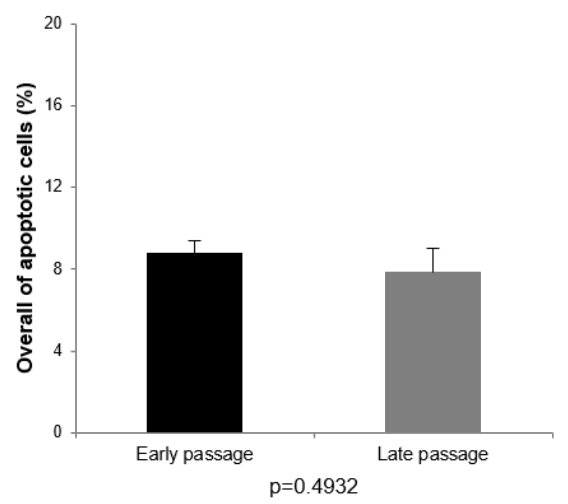

(D1)

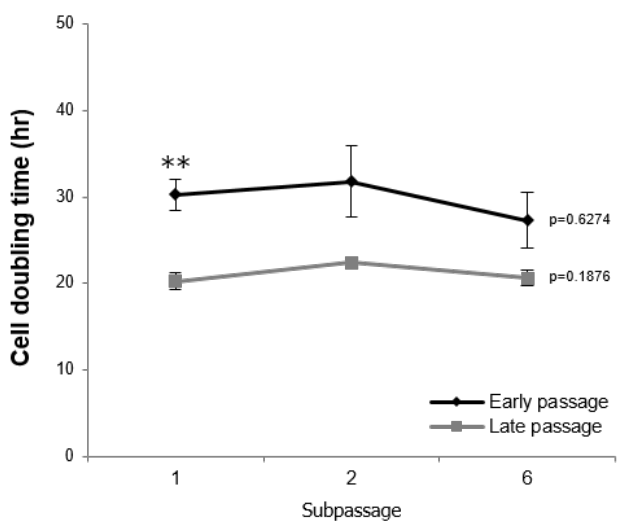

(A2)

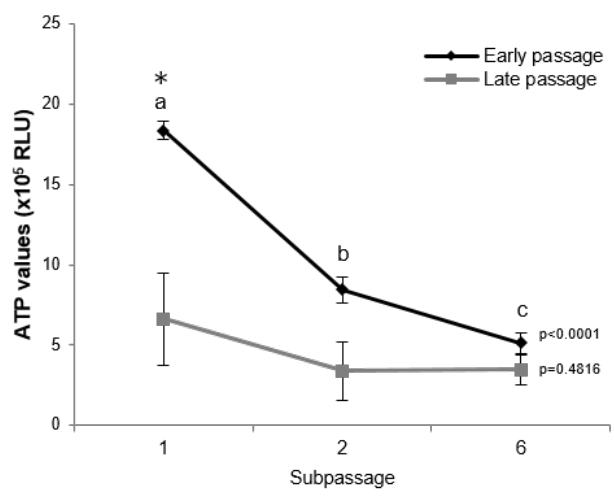

(B2)

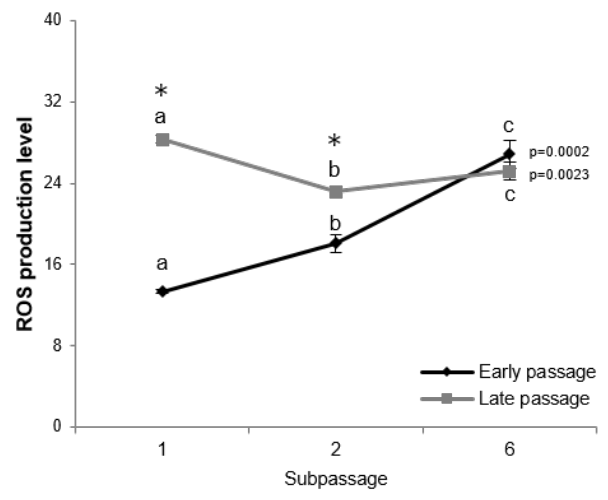

(C2)

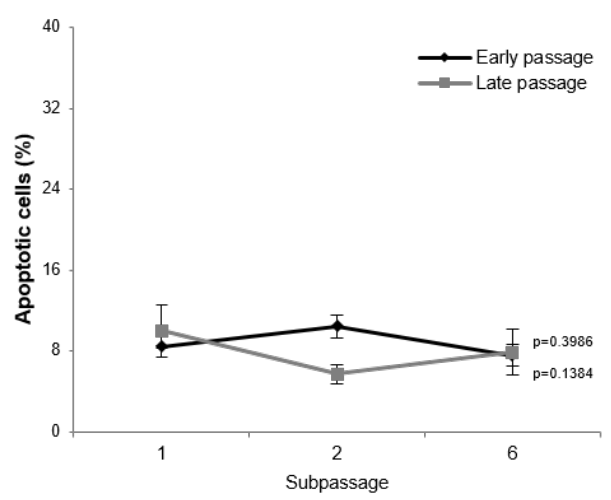

(D2)
Figure 2. (A) Proliferation of mouse embryonic stem cells (ESCs) subpassaged 4 (p4) or 19 times (p19) without cryopreservation. To monitor cell proliferation, doubling time of each cell line was calculated at the end of 1,2, and 6 subcultures after treatment. Accumulative levels of 1,2, and 6 subpassages (A1) and recording of each passage (A2). More proliferation was detected in the p19 ESCs than in the p4 ESCS, which was mainly derived from the difference at the first subculture. Within the same passage (p4 or p19 ESCs), the number of subpassges after treatment did not affect cell proliferation $(p=0.6274$ in the p4 ESCs and $p=0.1876$ in the p19 ESCs. (B) ATP synthesis in the p4 and p19 ESCs without cryopreservation. Synthetic activity of each ESCs was measured by luciferin-luciferase reaction at the end of 1, 2, and 6 subcultures after treatment. Accumulative levels of 1, 2, and 6 subpassages (B1) and recording of each passage (B2). More synthesis was detected in the p4 ESCS than in the p19 ESCs, which was mainly derived from the difference at the first subculture. In the p4 ESCs, ATP synthesis was significantly decreased as the number of subpassages $(p<0.0001)$. (C) Intracellular reactive oxygen species (ROS) levels in the p4 and p19 ESCs without cryopreservation. ROS levels in each ESC were assessed by FACS analysis using $2^{\prime}, 7^{\prime}$-dichloro-dihydro-fluorescein diacetate at the end of 1,2, and 6 subpassages after treatment. Accumulative levels of 1, 2, and 6 subpassages (C1) and recording of each passage (C2). Significant increase in ROS level was detected in the p19 ESCs than in the p4 ESCs. Within the same ESC passage, different pattern in ROS generation was detected as the passage was increased. (D) Proportions of the percentage of apoptotic cells to total p4 and p19 ESCs without cryopreservation. To monitor apoptotic cell numbers at the end of 1,2, and 6 subpassges, flow cytometry using FITC annexin $V$ was used. Accumulative levels of 1, 2, and 6 subpassages (D1) and recording of each passage (D2). No significant difference in the number was detected between p4 and p19 ESCs. Data are shown as mean \pm standard error. ${ }^{*} p<0.05$, * $p<0.01$. 


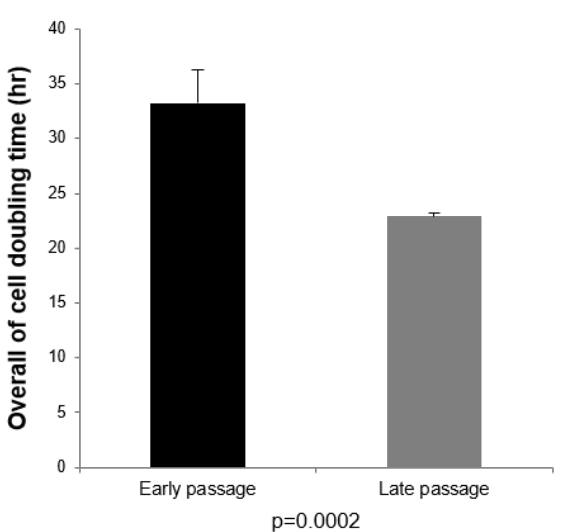

(A1)

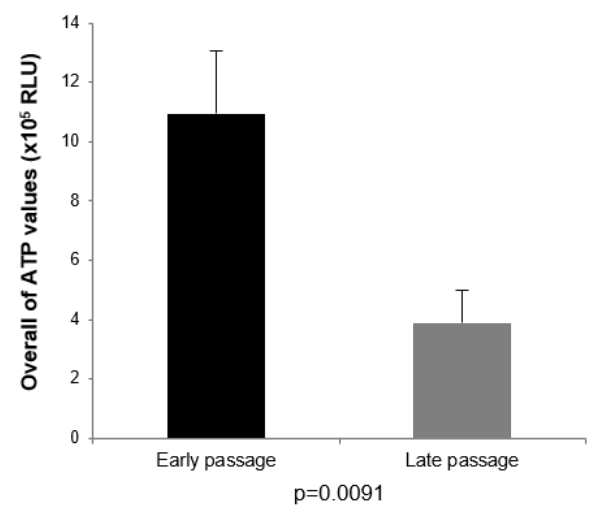

(B1)

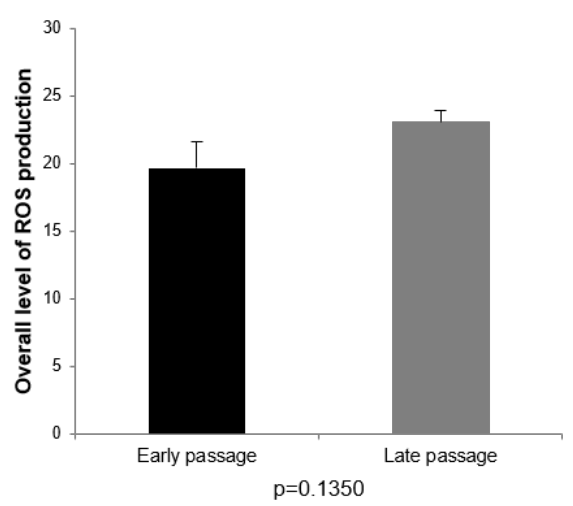

(C1)

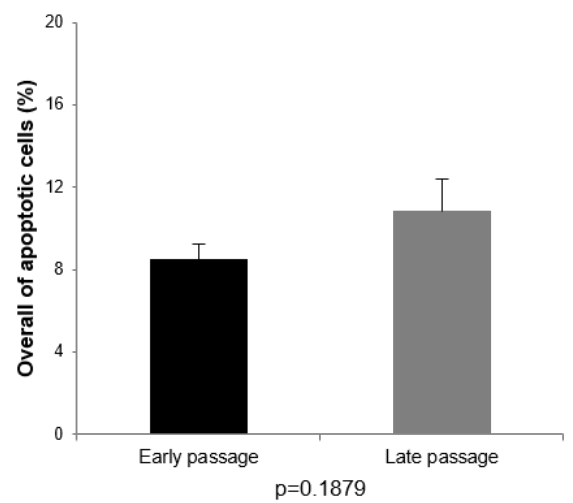

(D1)

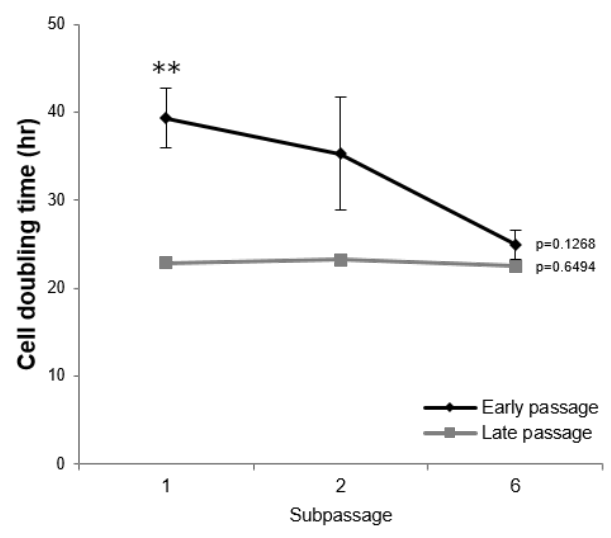

(A2)

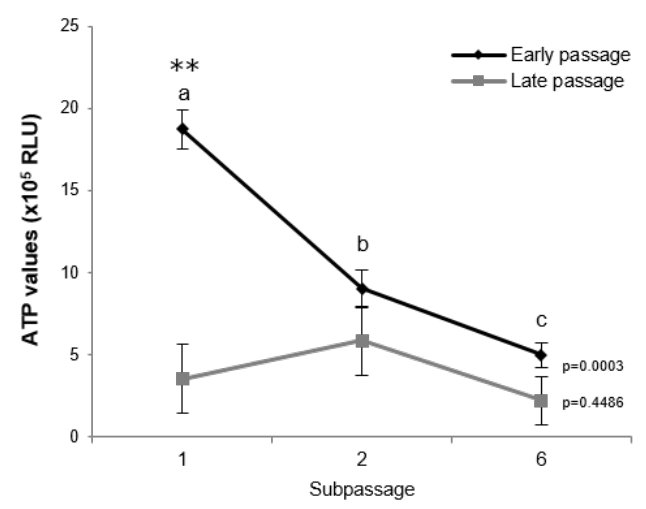

(B2)

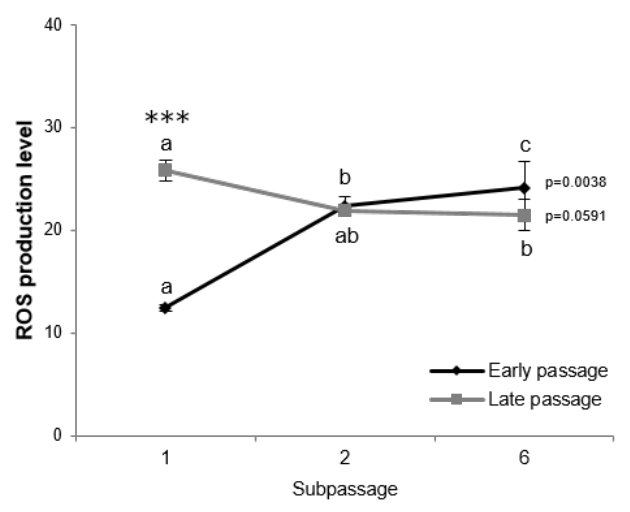

(C2)

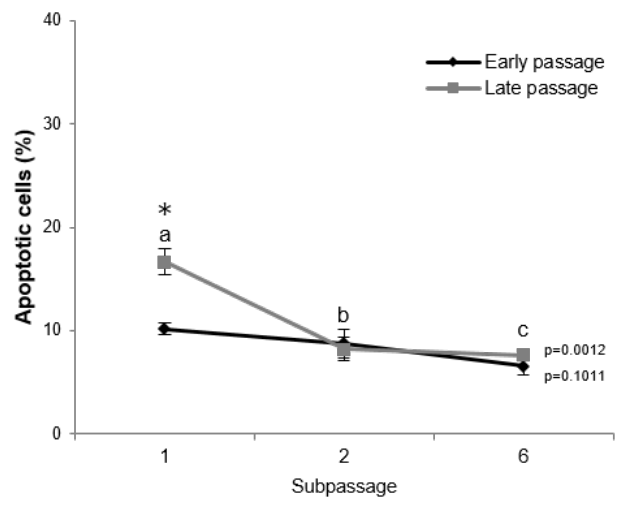

(D2)
Figure 3. (A) Proliferation of mouse embryonic stem cells (ESCs) subpassaged 4 (p4) or 19 times (p19) before cryopreservation. To monitor cell proliferation, doubling time of each cell line was calculated at the end of 1, 2, and 6 subpassages after cryopreservation. Accumulative levels of 1,2 , and 6 subpassages (A1) and recording of each passage (A2). More proliferation was detected in the p19 ESCs than in the p4 ESCs, which was mainly derived from the difference at the first subpassage after cryopreservation. Within the same passage (p4 or p19), the number of subcultures after cryopreservation did not affect cell proliferation ( $p=0.1268$ in the p4 ESCs and $p=0.6494$ in the p19 ESCs. (B) ATP synthesis in the p4 and the p19 ESCs before cryopreservation. Synthetic activity of each cell was measured by luciferin-luciferase reaction at the end of 1,2, and 6 subpassages after cryopreservation. Accumulative levels of 1, 2, and 6 subpassages (B1) and recording of each passage (B2). More synthesis was detected in the p4 ESCs than in the p19 ESCs, which was mainly derived from the difference at the first subpassage after cryopreservation. In p4 ESCS, ATP synthesis was significantly decreased as the number of subpassages was increased ( $p=$ 0.0003). (C) Intracellular reactive oxygen species (ROS) levels in the p4 and p19 ESCs before cryopreservation treatment. ROS levels in the ESCs were assessed by FACS analysis using $2^{\prime}, 7^{\prime}$-dichloro-dihydro-fluorescein diacetate at the end of 1,2, and 6 subpassages. Accumulative levels of 1, 2, and 6 subpassages (C1) and recording of each passage (C2). Within the same ESC passage, different pattern in ROS generation was detected as the passage after cryopreservation was increased, which yielded a significant difference $(p<0.0038)$ except for the group of late passage with cryopreservation. (D) Proportions of the percentage of apoptotic cells to total p4 and p19 ESCs before cryopreservation treatment. To monitor apoptotic cell numbers at 1, 2, and 6 subpassges, flow cytometry using FITC annexin $V$ was used. Accumulative levels of 1, 2, and 6 subpassages (D1) and recording of each passage (D2). No significant difference in the number was detected between the p4 and the p19 ESCs. Within the same ESC passage, significant difference was only detected in the p19 ESCs $(p=0.0012)$. Data are shown as mean \pm standard error. ${ }^{*} p<0.05,{ }^{* *} p<0.01$, ${ }^{* * *} p<0.001$. 
by cell banking system. From different viewpoint, subculture of ESCs under the same condition reduces batch effect due to the difference in passage number and at least twice subculture of the ESCs used in this study is recommended for yielding non-fluctuated data. Different culture regimen may require additional cell manipulation (subpassage) before undertaking main experimentation.

\section{CONFLICT OF INTEREST}

We certify that there is no conflict of interest with any financial organization regarding the material discussed in the manuscript.

\section{ACKNOWLEDGMENTS}

This research was supported by Bio-industry Technology Development Program (IPET312060-5), Ministry for Food, Agriculture, Forestry and Fisheries, Republic of Korea and by the National Research Foundation of Korea (NRF) grant funded by the Korea government (MSIP) (No. 2015R1C1A2A 01055746).

\section{REFERENCES}

1. Bardelli S, Moccetti M. Stem Cell banking and its impact on cardiac regenerative medicine. Adv Exp Med Biol 2016;951: 163-78.

2. Hilkens P, Driesen RB, Wolfs E, et al. Cryopreservation and banking of dental stem cells. Adv Exp Med Biol 2016;951:199235.

3. Woods EJ, Perry BC, Hockema JJ, et al. Optimized cryopreservation method for human dental pulp-derived stem cells and their tissues of origin for banking and clinical use. Cryobiology 2009;59:150-7.

4. Perry BC, Zhou D, Wu X, et al. Collection, cryopreservation, and characterization of human dental pulp-derived mesenchymal stem cells for banking and clinical use. Tissue Eng Part C Methods 2008;14:149-56.

5. Stacey G. Banking stem cells for research and clinical applications. Prog Brain Res 2012;200:41-58.

6. Sun C, Yue J, He N, et al. Fundamental principles of stem cell banking. Adv Exp Med Biol 2016;951:31-45.

7. Murphy A, McKenna D, McCullough J. Cord blood banking and quality issues. Transfusion 2016;56:645-52.

8. Vaidya A, Singhania S. Quality control measures in cord blood banking in India - critical appraisal and recommendations. J Stem Cells 2013;8:105-13.

9. Diaferia GR, Cardano M, Cattaneo M, et al. The science of stem cell biobanking: investing in the future. J Cell Physiol 2012;227:14-9.

10. Kim GA, Lee ST, Ahn JY, Park JH, Lim JM. Improved viability of freeze-thawed embryonic stem cells after exposure to glutathione. Fertil Steril 2010;94:2409-12.

11. International Stem Cell Banking I. Consensus guidance for banking and supply of human embryonic stem cell lines for research purposes. Stem Cell Rev 2009;5:301-14.

12. Ilic D, Ogilvie C. Human embryonic stem cells-what have we done? What are we doing? Where are we going? Stem Cells 2017;35:17-25.

13. Kadota S, Aiba K, Nakatsuji N. Embryonic stem cell research. Nihon Rinsho 2011;69:2109-13.

14. Nakamura Y. Bio-resource of human and animal-derived cell materials. Exp Anim 2010;59:1-7.

15. Healy LE, Ludwig TE, Choo A. International banking: checks, deposits, and withdrawals. Cell Stem Cell 2008;2:305-6.

16. Nottola SA, Albani E, Coticchio G, et al. Freeze/thaw stress induces organelle remodeling and membrane recycling in cryopreserved human mature oocytes. J Assist Reprod Genet 2016;33:1559-70.

17. Morgenstern DA, Ahsan G, Brocklesby M, et al. Post-thaw viability of cryopreserved peripheral blood stem cells (PBSC) does not guarantee functional activity: important implications for quality assurance of stem cell transplant programmes. $\mathrm{Br}$ J Haematol 2016;174:942-51.

18. Smagur A, Mitrus I, Giebel S, et al. Impact of different dimethyl sulphoxide concentrations on cell recovery, viability and clonogenic potential of cryopreserved peripheral blood hematopoietic stem and progenitor cells. Vox Sang 2013;104:240-7.

19. Woods EJ, Thirumala S, Badhe-Buchanan SS, Clarke D, Mathew AJ. Off the shelf cellular therapeutics: Factors to consider during cryopreservation and storage of human cells for clinical use. Cytotherapy 2016;18:697-711.

20.Pal R, Totey S, Mamidi MK, Bhat VS, Totey S. Propensity of human embryonic stem cell lines during early stage of lineage specification controls their terminal differentiation into mature cell types. Exp Biol Med (Maywood) 2009;234:1230-43.

21. Park YB, Kim YY, Oh SK, et al. Alterations of proliferative and differentiation potentials of human embryonic stem cells during long-term culture. Exp Mol Med 2008;40:98-108.

22.Enver T, Soneji S, Joshi C, et al. Cellular differentiation hierarchies in normal and culture-adapted human embryonic stem cells. Hum Mol Genet 2005;14:3129-40.

23. Maitra A, Arking DE, Shivapurkar N, et al. Genomic alterations in cultured human embryonic stem cells. Nat Genet 2005;37:1099-103.

24. Huang HL, Hsing HW, Lai TC, et al. Trypsin-induced proteome alteration during cell subculture in mammalian cells. J Biomed Sci 2010;17:36.

25.Li Y, Ma T. Bioprocessing of cryopreservation for large-scale banking of human pluripotent stem cells. Biores Open Access 2012;1:205-14.

26. Miki T, Wong W, Zhou E, et al. Biological impact of xeno-free chemically defined cryopreservation medium on amniotic 
epithelial cells. Stem Cell Res Ther 2016;7:8.

27.Sart S, Ma T, Li Y. Cryopreservation of pluripotent stem cell aggregates in defined protein-free formulation. Biotechnol Prog 2013;29:143-53.

28. Kim GA, Kim HY, Kim JW, et al. Ultrastructural deformity of ovarian follicles induced by different cryopreservation protocols. Fertil Steril 2010;94:1548-50.e1.
29. Wagh V, Meganathan K, Jagtap S, et al. Effects of cryopreservation on the transcriptome of human embryonic stem cells after thawing and culturing. Stem Cell Rev 2011;7:506-17.

30.Li XY, Jia Q, Di KQ, et al. Passage number affects the pluripotency of mouse embryonic stem cells as judged by tetraploid embryo aggregation. Cell Tissue Res 2007;327:607-14. 